\title{
Piperine Extract (Standardized)
}

National Cancer Institute

\section{Source}

National Cancer Institute. Piperine Extract (Standardized). NCI Thesaurus. Code C48417.

A standardized extract containing the active alkaloid piperine, derived from the fruit of the plant Piper nigrum (black pepper) and/or the plant Piper longum L. (long pepper), with thermogenic properties. Co-ingestion of piperidine enhances the bioavailability of various nutrients, including beta-carotene, curcumin, selenium, pyridoxine and coenzyme Q10. In addition, this agent may exert anti-inflammatory and anti-tumor activities and may enhance the production of serotonin. 